\title{
PWM Technique To Overcome The Effect of High PAPR in Wireless Systems
}

\author{
Omar Daoud \\ Department of Communications and Electronics Engineering, Philadelphia University, \\ Amman, Jordan
}

\begin{abstract}
Many current communication systems suffer from performance degradation due to the high sensitivity to high power peaks especially in the nonlinear devices. The author introduces a new concept based on the Pulse Width Modulation (PWM), namely MIMO-OFDM system based PWM (MO-PWM) to overcome this deficiency. Here, the peak-to-average power ratio (PAPR) problem in Orthogonal Frequency Division Multiplexing (OFDM) technique is used as a criterion to check the validity of the proposed work. Moreover, the proposed system work has been implemented over Field Programmable Gate Array (FPGA), which is designed to characterize both of the complexity and the speed issues.
\end{abstract}

The systems performance based MO-PWM and validity have been checked based on a numerical analysis and a conducted simulation. The simulation results show that the MO-PWM can clearly reduce the PAPR values nevertheless the used OFDM systems' specifications, and gives a promising results over some techniques found in the literature, such as clipping, SLM and PTS under same bandwidth occupancy and system's specifications.

\section{KEYWORDS}

MIMO, OFDM, Peak-to-Average Power Ratio, wavelet, Eigen vectors component.

\section{INTRODUCTION}

Based on the ever-growing demand for wireless systems' users which leads to the fact that so many systems has been rise in the last decade; such as WiFi, WiMax, HSPA, LTE, Bluetooth-2 [1-6]. Thus and to meet the needs of having a high data rates for such applications, Orthogonal Frequency Division Multiplexing (OFDM) technique has been imposed. Its based on transmitting data simultaneously over equally spaced carrier frequencies [1,2]. The main Blocks that are used to produce the OFDM signal are the Fast Fourier Transform (FFT) and its Inverse (IFFT), which will give its importance in achieving high speed processing by performing the transformation process, filtering and correlation with fewer operations[7-9]. However, there are some drawbacks that could affect its performance such the need to high dynamic range in the nonlinear devices and the synchronization issues. This work will focus on the Peak-to-Average Power Ratio (PAPR), which expresses the high dynamic range problem. This is due to that it increases the cost of such devices; Radio Frequency (RF) power amplifiers, mixers and analogue to digital converters $[1,3]$. Therefore, average signal power must be kept low in order to prevent the transmitter amplifier and other circuitry limiting. Minimizing the PAPR allows a higher average power to be transmitted for a fixed peak power, which leads to improve the overall signal to noise ratio at the receiver. In order to have the remedy for such drawback, there are so many solutions in the literature to tackle this issue, such as Selective mapping, Golay sequences, Cyclic coding, clipping and filtering; and multiple signal representation techniques [10-12]. 
The work in this paper is divided into two main parts; the first contains the implementation process of OFDM transceiver on FPGAs. Despite the fact that the most of existing work has been accomplished on either speed or area, this work kept in mind the urgent need to optimize both of them all together. Therefore, an optimum solution will be provided for today's wireless communication systems. The other one has a new proposition for combating the effect of the PAPR. The proposed algorithm tackles the PAPR using the Pulse Width Modulation (PWM) technique, which is considered as the basis for control in power electronics. This is due to its waveform representation in driving and controlling the power devices [13].

\section{Pulse Width Modulation}

The PWM signal could be defined as pulses with variable widths that are determined by the input signal levels. Mathematically, the binary output format could be written as [13]

$$
\operatorname{PWM}(\mathrm{t})=\operatorname{sgn}(\mathrm{r}(\mathrm{t})-\mathrm{c}(\mathrm{t})) \text {, }
$$

'sgn' is the sign function, $\mathrm{r}(\mathrm{t})$ is the compared reference signal and $c(t)$ is the carrier signal. Two basic methods can be used to generate the PWM signals; namely the PWM direct digital generation and the uniformly sampled PWM. In direct digital generation method, the value of the modulated signal is key value that controls the width of the PWM pulse, where in the uniformly sampled version the $c(t)$ can be generated by a Digital Signal Processing (DSP) clock signal. Moreover, three commonly carrier signals could be used and generated a constant frequency PWM signal; namely Sawtooth, Inverted Sawtooth, and Triangle carriers. In our case, a Triangle $c(t)$ has been chosen since it has much less dominant higher harmonics. This is in order to reduce the needed system bandwidth, which is related to the found higher harmonics number. This is in addition to that the uniformly sampled PWM does not require a high frequency clock signal [13, 14]. Figure 1 depicts the uniformly sampled PWM block diagram.

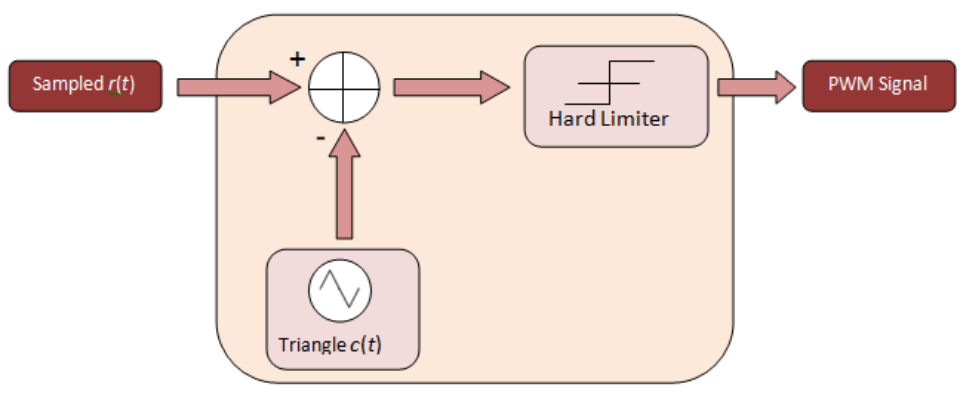

Fig. 1. Uniformly sampled PWM block diagram

This part of the system could be easily implemented to the used FPGA since the generated triangular wave comes from using a simple integration process applied to the squared wave clock signal. The hard limiter represented by a kind of comparator stage between the applied input sampled signal and the generated triangular signal.

\section{Peak-to-Average Power Ratio}

[15] Clearly describe the process of generating the OFDM signals from the inherent Fourier transform operation. Due to the addition process with different frequencies and phases of numerous waves, the high Peak-to-Average Power Ratio problem arises. As results, the transmitter with high dynamic ranges should be used to overcome such deficiency. Hence, it has turned the researchers' attention toward how to optimize and propose solution at the expense of 
the computational complexity, the bit error rate (BER) degradation, or the side information transmission process (SI) that decreases the spectral efficiency, such as amplitude clipping, selective mapping (SLM), partial transmit sequence (PTS), coding based schemes, shifted phase sequence techniques, transformation based techniques and tone based schemes [15-18].

Generally, the predicted PAPR in the OFDM signal can be easily written as:

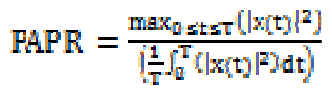

Here, $x(t)$ is the transmitted OFDM symbol that is generated by modulating a data block of $\mathbf{N}$ symbols; $\mathrm{x}_{\mathrm{K}}$ by a set of orthogonal subcarriers; $\mathrm{f}_{\mathrm{k}}$ forl $=0, \ldots, \mathrm{N} \quad 1$ and $\mathrm{T}$ is the duration of the OFDM symbol that can be used to maintain the orthogonality among the OFDM subcarriers; $0 \leq t \leq T$. Thus, it can be clearly defined as:

$$
x(t)=\frac{1}{* \mathbb{N}}\left(\sum_{k=0}^{N-1}\left(X_{k}, e^{\left(j z \pi f_{k} \pm j\right)}\right)\right)
$$

However, and as mentioned in [19], it can be generated and implemented practically using the IFFT. In this situation, the oversampling process should be taken into consideration. Therefore (2) is modified using the over-sampling factor Wand a discrete time version of $x(t)$ as follows:

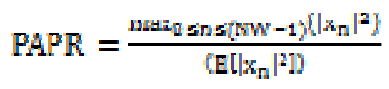

$\mathrm{n}$, is the number of samples, which is oversampled by $W$, and $\mathbb{E}[$ ] ]is the expectation value.

In this work, a new proposition for an algorithm based PWM has been suggested and justified. Moreover, the proposed work has been compared with conventional OFDM systems in order to show the performance improvements as will shown in section 3.

This work is divided into four sections. In section II, the proposed scheme that is based on PWM is introduced along with the analytical formulation in addition to the computational complexity. Section III presents simulation results and hardware implementations, followed by the concluding remarks in Section V.

\section{OFDM system-based PWM}

In this subsection a new algorithm has been proposed to allocate the high peaks that found in an OFDM signal. As shown in Figure 1, the flowchart explains the following procedure to attain the target from the proposed algorithm. Thus, the proposed algorithm starts with scan the produced OFDM signal after the Fast Fourier Transform process (FFT)

This section depicts the proposition of an efficient designed technique for overcoming the high PAPR deficiency in the OFDM systems. Figure 2 shows the overall proposed OFDM system based PWM block diagram, where the position of the proposed work will be placed after the Inverse-FFT (IFFT) stage.

As shown in Figure 2, the proposition is based on processing the generated OFDM symbol after the IFFT block by a PWM. It is inserted after a completed OFDM symbol, i.e. after the guard interval (GI) that is generated by making use of the cyclic prefix (CP) process insertion process. This is in order to reduce the expected complexity by reducing the resultant inter symbol interference (ISI).

In this section, an efficient technique for reducing the effect of PAPR will be illustrated. By 
imposing the PWM block, the system performance should be enhanced; maximum power amplifier efficiency issues attained in addition to the ability of using the nonlinear devices. This is true for the achieved almost 0 complementary cumulative distribution function (CCDF) curves. Figure 3 depicts the procedure of the proposed work. It starts with scanning the resultant $x(n, m)$, which is drawn from (3) and shown in (5) to be processed as shown in Figure 3.

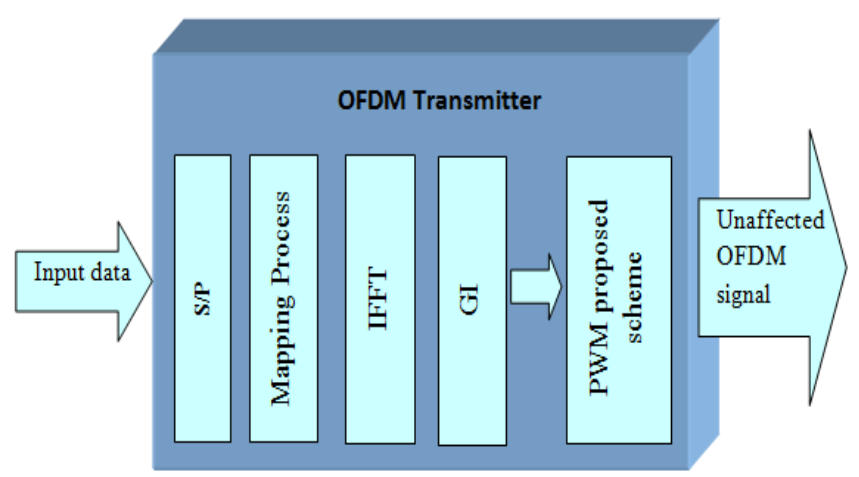

Fig. 2. OFDM-based PWM transmitter block diagram

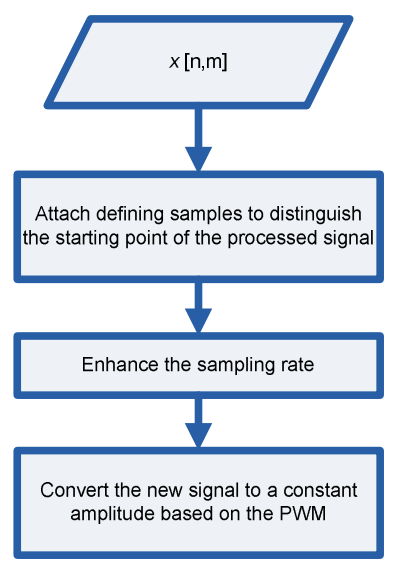

Fig.3. The conversion procedure flowchart

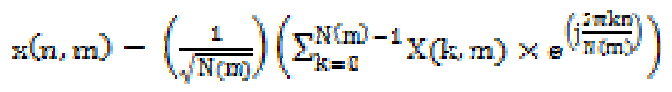

Here, the block length is defined by $\mathrm{N}(\mathrm{m})$ as the number of IFFT points at time index (Block index) $m$ which takes either 0 or $\mathrm{j}$ as $\mathrm{m}=\left\{\begin{array}{cc}0, & \mathrm{n}<N(0) \\ \mathrm{j}, & \sum_{\mathrm{i}=0}^{j-1} \mathrm{~N}(\mathrm{i}) \leq \mathrm{n}<\sum_{\mathrm{i}=0}^{\mathrm{j}} \mathrm{N}(\mathrm{j}), \mathrm{j} \geq 1\end{array}\right.$.

In the proposed PAPR reduction method, a new block based PWM is inserted as shown in Figure 2. It could be inserted after the cyclic prefix (CP) block to ensure having a free of ISI channel. Moreover, to offer a perfect circular convolution it preferred to be added before the CP block. Therefore, the insertion choice will be optimized to fit the cost and complexity equation criteria. In this work, it has been inserted the $\mathrm{CP}$ block to reduce the hardware area that will be allocated in the used FPGA.

The new proposed algorithm to overcome the effect of the OFDM signal high peaks in this section will be processed as follows and it will be summarized as shown in Figure 4: 
International Journal of Computer Networks \& Communications (IJCNC) Vol.6, No.4, July 2014

\section{The pre-process stage:}

- Attach a zero sample to lead the OFDM symbol as a distinguishing one. Therefore, the start point of each symbol will be known.

- Enhance the conversion accuracy by increasing the number of samples between the adjacent OFDM samples; $\hat{k}(n, m)$ with a new sampling rate; $\hat{N}(m)$.

- Convert $\hat{k}(\mathrm{n}, \mathrm{m})$ from vector into a matrix with three different vectors as $\hat{\mathrm{k}}(\mathrm{I}, \mathrm{i})=\left\{\begin{array}{c}\hat{\mathrm{z}}(\mathrm{n}, \mathrm{m}) \\ \hat{\mathrm{Z}}(\mathrm{n}, \mathrm{m})-\frac{1}{1+\mathrm{N}(\mathrm{m})} \\ \hat{\mathrm{N}}(\mathrm{n}, \mathrm{m})+\frac{1}{1+\mathrm{N}\left(\mathrm{m}^{2}\right.}\end{array}\right.$
1 to $(\hat{\mathrm{N}}(\mathrm{m})+\mathrm{N}(\mathrm{m}))$.

\section{The Comparison Stage:}

- Each sample inside $\hat{x}(\hat{j})$ will be compared with $\hat{x}(1-1)$ and modified to have constant amplitude using the following formula:

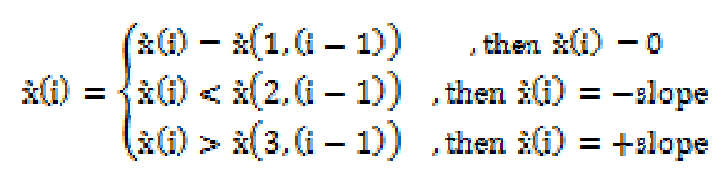


International Journal of Computer Networks \& Communications (IJCNC) Vol.6, No.4, July 2014

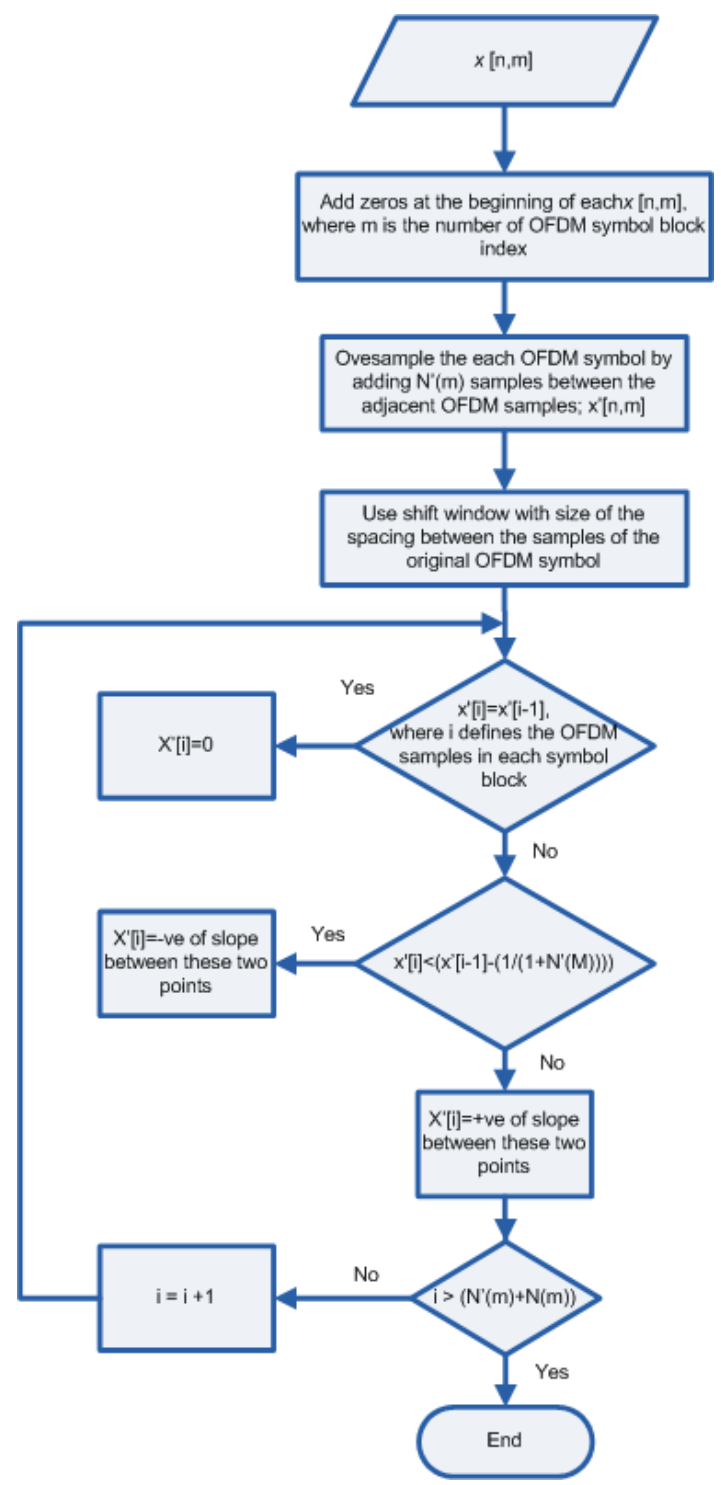

Fig. 4. The proposed scheme flowchart

Figure 5 depicts the proposed work structure. Thus, it is clearly shown the idea behind this work; any OFDM signal will be transferred to have constant envelope. It is clearly shown from Figure 5 that the OFDM symbol is transformed to a constant amplitude symbol and then the PAPR issues will not appear. Moreover and for simplicity the hermitian OFDM system should be used [20]. 


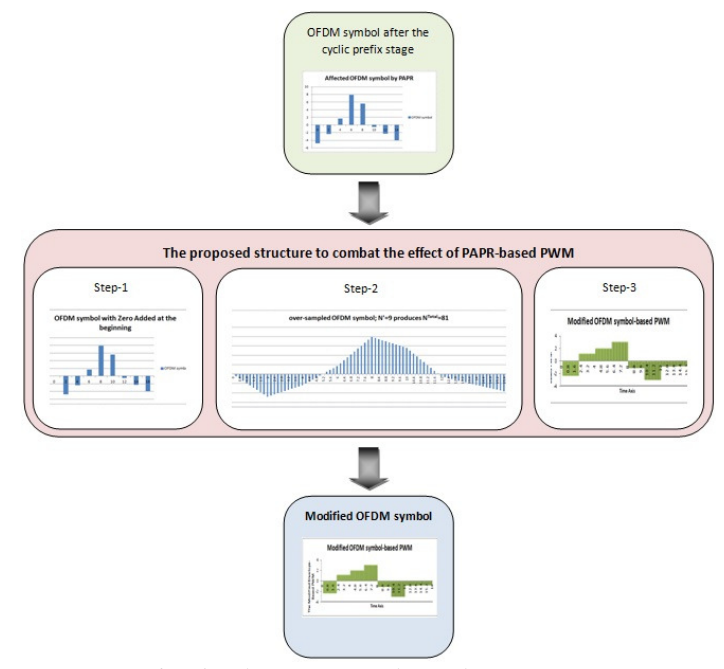

Fig.5. The proposed work structure

In the receiver side, the regeneration of the OFDM symbol could be done using the following procedure:

\section{1) Reconversion stage:}

- The original sample will equal the previous one if a zero sample is received.

- The original sample will equal the previous sample added a certain value with respect to the slope criterion calculation between the original sample and the previous received sample.

\section{2) De-processing stage:}

- Under sampling the resultant signal from step 1; the sampling rate will be reduced to $\mathrm{N}(\mathrm{m})$.

- Removing the zero-sample prefix from the OFDM symbol. Furthermore, for simplicity, this procedure is depicts in Figure 6.

Figure 2 represents the specification of the proposed MIMO-OFDM system based PWM (MOPWM) block diagram. The MO-PWM system consists of three main parts; OFDM transmitter, the proposed algorithm and the MIMO transmitter. For the OFDM stage; the system model will be limited to the use of simple convolutional encoder, Quadrature Shift Keying (QPSK) mapping techniques, 256-IFFT points and 0.25 CP lengths. As a result the OFDM symbol will be used as an input to enter the second part; proposed work. As mentioned earlier, the high peaks could appear after the FFT stage, where OFDM symbols were added coherently at the same phase. Thus, the second stage will convert any OFDM symbol to a PWM signal with peaks of $\pm 0.5 \mathrm{~V}$. The processed signal will be sent through the Vertical-Bell Laboratories Layered Space-Time (VBLAST) MIMO system.

This technology has been proposed to enhance the system capacity/throughput expressed in terms of bits/symbol. Moreover, the Guard Interval (GI) block is used to add the cyclic prefix interval to combat the effect of either inter symbol interference (ISI) and/or inter carrier interference (ICI). The proposed work novelty rises from the way of dealing with the OFDM symbol, where the CCDF curves are guaranteed to remains at zero level. Moreover, and in order to check the validity of the proposed work, MO-PWM has been designed and built over a FPGA technology. 
International Journal of Computer Networks \& Communications (IJCNC) Vol.6, No.4, July 2014

Some issues have been taken into consideration, such as hardware speed and timing simulation.

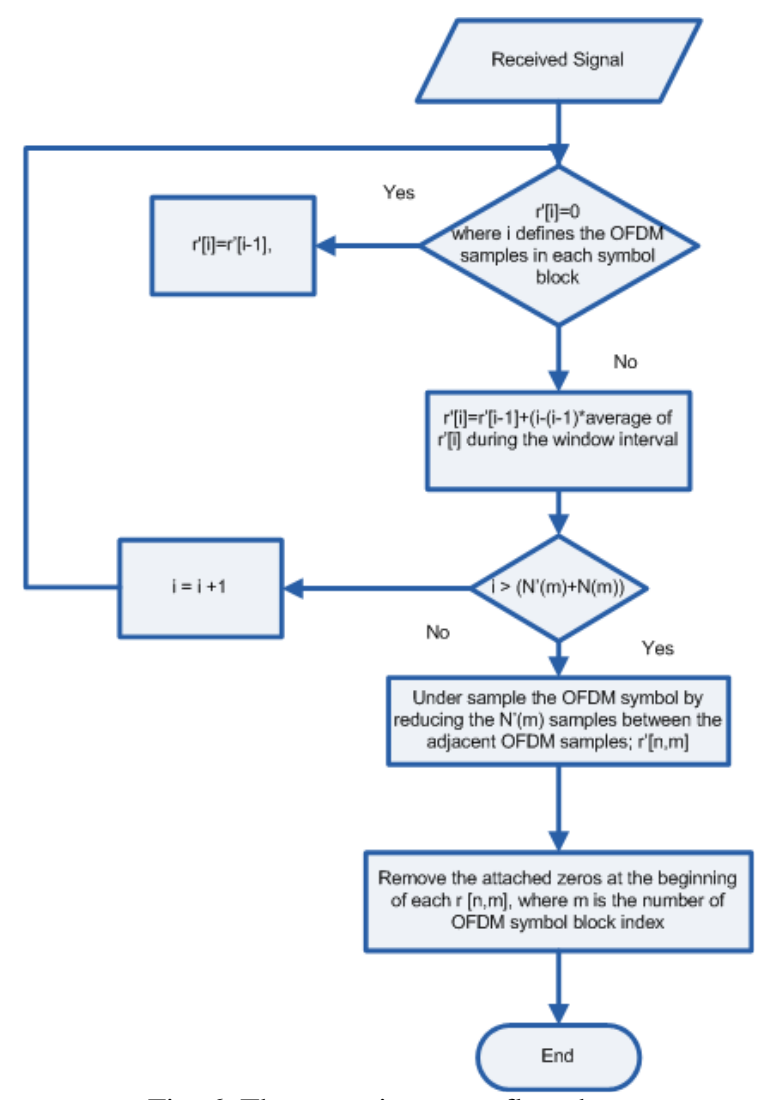

Fig. 6. The reception stage flowchart

The next section describes the results from the simulation of the proposed work against the conventional techniques.

\section{MO-PWM SYSTEM TESTED RESULTS}

A WiMAX MO-PWM system's performance has been studied through a performed MATLAB simulation and a verilog-HDL based hardware programmed FPGA. The first part contains the system's throughput considerations, while the other part handles the hardware implementation. For the simulation area, the channel bandwidth has been selected to be $20 \mathrm{MHz}$. For the oversampling stage; the $N^{\prime}(m)$ value has two different values 9 , and 63. For the hardware part, the hardware has been implemented over the available Xilinix XC2S150, one of the Spartan 2E series. This is in addition to the use of ISE tool chain, which shows the behavioral test and the synthesis of the hardware block diagram [21].

\section{Simulation Part}

Based on MATLAB, Figure 7 shows the simulated system parts with the previously mentioned specifications. The proposed work is twice inserted to work on the real OFDM symbol and cover the real and imaginary parts. 


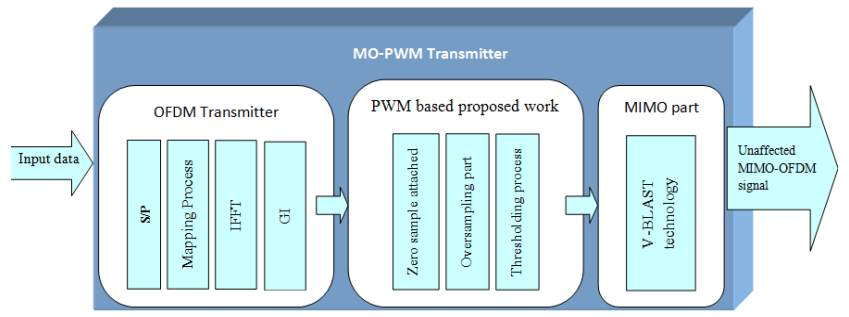

Fig. 7. MO-PWM system transmitter part

This part shows the performance of the proposed work in terms of the sample error rate (SER). Figure 8 depicts the achieved SER results of the proposed work which is equal to $39.67 \times 10^{-4}$. It is a promising achieved value for the MO-PWM work. Furthermore and in order to enhance this result and under the cost of increasing the delay time, a modification has been made in the recovery process and we impose the average of the received signals during the window time instead of using the previous received sample. This modification increases the SER to be $38.63 \times 10^{-4}$ instead of $39.67 \times 10^{-4}$.

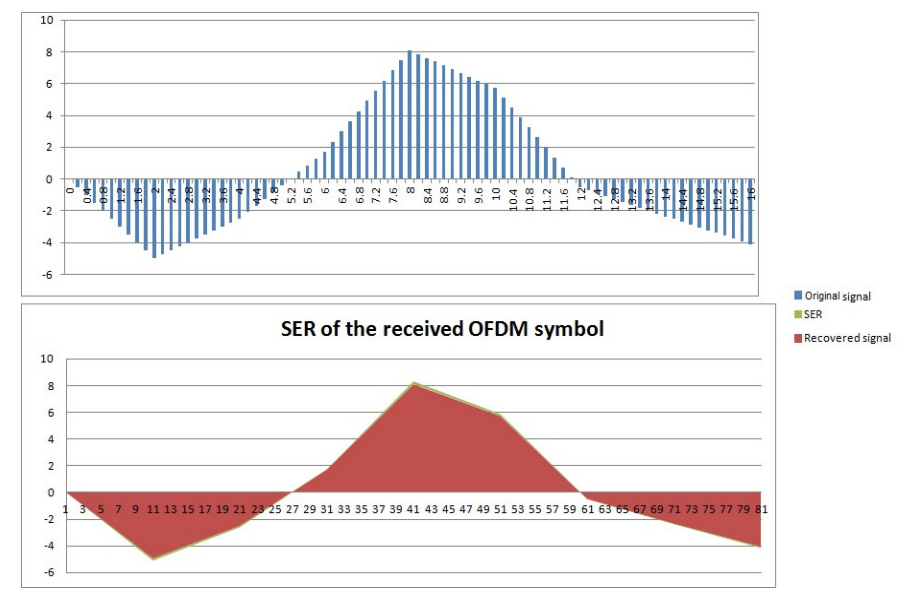

Fig. 8. The SER of the MO-PWM work

Furthermore and to check the CCDF curves of the MIMO-OFDM systems performance; Figure 9 shows the CCDF curves and the achieved enhancement over the conventional OFDM CCDF curves. A s expected and compared to as an example the ordinary WiMax which has a range of 8.5-14.5 dB PAPR, the large peaks that could be found in the MO-PWM have been reduced from $10^{-4}$ to be around $10^{-13}$ which is almost 0 at $20 \mathrm{~dB}$ threshold.

Based on the simulation results shown in Figures 8 and 9, it is clearly shown that the MO-PWM work has improves the PAPR values and completely overcome its effect. This is true under the cost of $38.63 \times 10^{-4}$ SER ratio in the recovery process. In order to increase the system efficiency, another factor has been taken into consideration to be studied; the effect of extra inserted samples. Here, since the MO-PWM works on sending the slope value of the samples during a window instead of the original values, the number of inserted samples does not matter and has no effect like the work in the literature on the SER. 


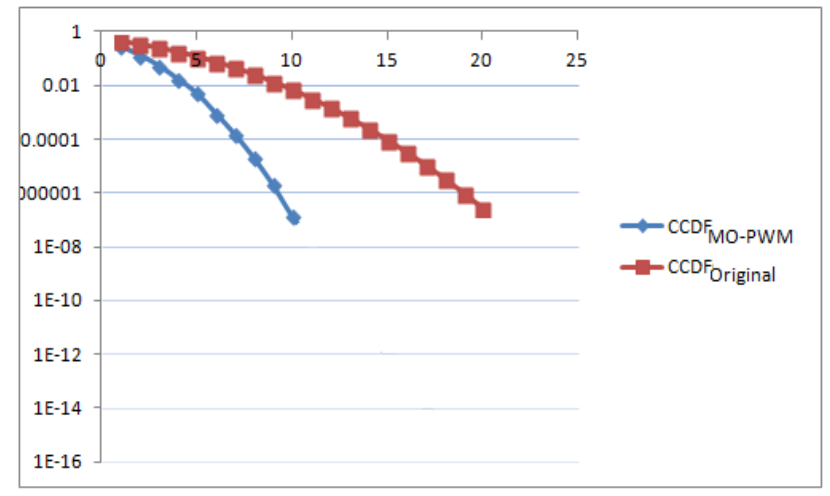

Fig.9. CCDF results based on QPSK modulation technique

Moreover, the MO-PWM is different from the conventional work in the literature that is based on either delta modulation or fixed constant amplitude. This is because delta modulation has two cases of comparison and fixed values, while MO-PWM links the comparison stage with the slope of the OFDM samples during a window, which has the size of the separation interval between two adjacent samples in the original OFDM symbol. Additionally, the continuous amplitude PWM has three comparison cases and links them with the number of inserted samples. It has to be high number of inserted samples to enhance the system efficiency, which MO-PWM is not and there is no links between the SER and the number of inserted extra samples. For future work, a room of comparison with techniques found in the literature such as Partial Transmit Sequence (PTS), Selective Mapping (SLM) or Clipping techniques or our previously published work is available. This will be based on the simplicity, delay time, cost, complexity and the transmission throughput issues.

\section{Hardware Implementation Part}

In this work and to check the feasibility of the MO-PWM, it has been built in accordance to the WiMax specifications. The algorithm was implemented in the available FPGA Xilinix card XC2S150 [21]. MO-PWM system shown in Figure 7 is decomposed into its functional blocks to be implemented using the verilog; a well known hardware description language (HDL). Furthermore, the ISE behavioral simulator has been used to test and evaluate each block simultaneously. From the generated ISE report, which shows the device utilization, an approximately $70 \%$ from the device were used. This is in addition to making use of the ModelSim EDA tool to accomplish the behavior and timing simulation [22]. From the hardware speed report, it is clearly shown that the MO-PWM reduces the use of maximum clock frequency up to $50 \%$, while the overall execution time is about $9.3 \mu$ s. Table 1 shows the report summary for MOPWM transmitter's and receiver's parts. 
International Journal of Computer Networks \& Communications (IJCNC) Vol.6, No.4, July 2014

Table 1 . The synthesis and place and route summary reports

\begin{tabular}{|l|l|l|}
\hline \multirow{2}{*}{} & \multicolumn{2}{|c|}{ MO-PWM } \\
\cline { 2 - 3 } & Transmitter side & Receiver side \\
\hline \multicolumn{3}{|c|}{ Place and route report } \\
\hline Number of slices & 78 out of 13696 & 15 out of 13696 \\
\hline Number of IOs & 18 out of 566 & 18 out of 566 \\
\hline \multicolumn{3}{|c|}{ Synthesis report } \\
\hline Number of slices & 95 out of 13696 & 8 out of 13696 \\
\hline Number of IOs & 18 out of 566 & 18 out of 566 \\
\hline $\begin{array}{l}\text { Number of GCLKs } \\
\text { Number of } 4 \text { input } \\
\text { LUTs }\end{array}$ & 166 out of 16 & 1 out of 16 \\
\hline
\end{tabular}

\section{Conclusions}

This work presents a new proposition to overcome the effect of high PAPR problem in OFDM systems, namely MO-PWM. The MO-PWM is based on transforming the OFDM signal into constant amplitude ones by making use of the slope between the consecutive samples.

To check the validity and feasibility of the MO-PWM, a MATLAB simulation in addition to a hardware implementation have been prepared and checked the validity of the proposed work analytical model.

This simulation is divided into two main parts; SER and CCDF curves, while the hardware has been used to check the hardware speed and area report. A comparison with the conventional work has been made and the MO-PWM enhancement has been exposed clearly in the previous section. A SER has been achieved around a $39.67 \times 10-4$. In a comparison with the conventional WiMAx systems, the PAPR ratio that may exceed certain threshold has been reduced from almost 10-4 to be almost 0 . Furthermore, and from the ISE behavioral report the MO-PWM has better execution time and enhance the uses clock frequency ranges.

For future work, a room of comparison with techniques found in the literature such as PTS, SLM or Clipping techniques or with our previously published work is available. This comparison may be made to tackle the simplicity, delay time, cost, complexity and the transmission throughput issues.

\section{REFERENCES}

[1] S. Hara and R. Prasad, Multicarrier Techniques for 4G Mobile Communications. Artech House, 2003.

[2] R. Prasad, OFDM for Wireless Communications Systems. Artech House, Inc., 2004.

[3] J. G. Andrews, A. Ghosh, and R. Muhamed, Fundamentals of WiMAX : Understanding Broadband Wireless Networking, T. S. Rappaport, Ed. Pearson Education, Inc., 2007.

[4] 3GPP, Tech. Specif. Group Services and System Aspects Service Requirements for Evolution of the 3GPP System (Rel. 8), 3GPP TS 22.278.

[5] E. Dahlman et al., 3G Evolution: HSPA and LTE for Mobile Broadband, 2nd ed., Academic Press, 2008.

[6] S. Abeta, "Toward LTE commercial launch and future plan for LTE enhancements (LTEAdvanced)," in Proc. IEEE International Conference on Communication Systems (ICCS), Singapore, pp. 146-150, 2010. 
[7] S. Parkvall, A. Furuskär, and E. Dahlman, "Evolution of LTE toward IMT-Advanced," IEEE Communications Magazine, pp. 84-91, Feb. 2011.

[8] Ahmed Saeed, M. Elbably, G.Abdelfadeel, "Efficient FPGA implementation of FFT/IFFT Processor" International Journal of circuits, systems and signal processing, Vol.3, Issue 3, pp. 103-110, 2009.

[9] P. Jackson, C. Chan, C. Rader, J. Scalera, and M. Vai. "A systolic FFT architecture for real time FPGA systems" High Performance Embedded Computing Conference (HPEC04), Sept. 2004.

[10] Y. Wang and Z. Luo, "Optimized Iterative Clipping and Filtering for PAPR Reduction of OFDM Signals”, IEEE Transaction on Communications, vol. 59, no. 1, pp. 33-37, 2011

[11] Chen Jung-Chieh, "Partial transmit sequence for PAPR reduction of OFDM signals with stochastic optimization techniques", IEEE Transactions on Consumer Electronics, vol. 56, no. 3, pp. 1229-1234, 2010.

[12] Sohn Insoo, "A Low Complexity PAPR Reduction Scheme for OFDM susyems via Neural Networks”, IEEE Communications Letters, vol. 18, no. 2, pp. 225-228, 2014.

[13] Francesco Vasca and Luigi Lannelli (Edit.), "Dynamics and Control of Switched Electronic Systems: Advanced Perspectives for Modelling, Simulation and Control of Power Converters", Springer Publisher, 2012.

[14] M. Koyuncu, C. van den Bos and W.A. Serdijn, "A PWM modulator for wireless infrared communication", Proceedings of the ProRISC/IEEE Workshop on Semiconductors, Circuits, Systems and Signal Processing, Veldhoven, The Netherlands, 30 Nov - 1 Dec, 2000, pp: 351-353, ISBN 9073461-24-3

[15] Tao Jiang and Yiyan Yu, "An overview: Peak-to-Average Power ratio Reduction Techniques for OFDM Signals”, IEEE Transaction on Broadcasting, vol. 54, no. 2, pp. 257-268, 2008

[16] C. Dick and H Tarn, "FPGA Realization of Peak-to-Average Power Reduction technique for OFDM wireless systems”, IEEE 41 Asilomar Conference on Signal Systems and Computers (ACSSC 2007), pp. 1969-1975, Pacific Group, California 2007.

[17] Seung Hee Han, and Jae Hong Lee, "An overview of peak-to-average power ratio reduction for multicarrier transmission”, IEEE wireless communication, vol. 12, no. 2, pp. 56-65, 2005.

[18] Luging Wang and C Tellambura, "An overview of peak-to-average power ratio reduction techniques for OFDM systems", IEEE international symposium on signal processing and information technology, pp. 840-845, Canada 2006

[19] Nee V. and Prasad R., "OFDM wireless multimedia communications", Artech House Boston London 2000.

[20] Hao LIN and Pierre SIOHAN, "OFDM/OQAM with Hermitian Symmetry: Design and Performance for Baseband Communication”, IEEE International Conference on Communications, (ICC '08), pp. 652-656, Beijing 2008.

[21] Xilinx-manufacturer of FPGA silicon and development tools, http://www.xilinx.com

[22] EDA tool, http://www.mentor.com

\footnotetext{
Authors

Omar Daoud (odaoud@philadelphia.edu.jo) has achieved the PhD in the field of Communication and Electronics Engineering at DMU/ UK 2006. He joined Philadelphia University in 2007 as Assistant Professor. His current work is about achieving the Quality of Service for the 4th Generation of the Wireless and Mobile Communication Systems by combining the advantages of the OFDM and the multiple antenna technology. He is the Assistant Dean in the Faculty of Engineering in addition to the Head of Communications and Electronics engineering department. Moreover, and in March 2012 he has promoted to the associate professor rank.
}

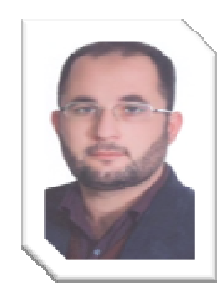

